\title{
Large colorectal mucosal defect closure post-endoscopic submucosal dissection using the reopenable clip over line method and modified locking-clip technique
}

Mucosal defects during extensive colorectal endoscopic submucosal dissection (ESD) cause post-ESD bleeding [1], and a method to close these defects has not yet been established. In recent years, the usefulness of a method for closing mucosal defects after colorectal ESD using a line and clips has been reported [2]. Therefore, we invented a new clip-line closure method called the "reopenable clip over line method" (ROLM), which uses a line $(0.23-\mathrm{mm}$ nylon line) and reopenable clips (SureClip; 8 or $16 \mathrm{~mm}$; MC Medical, Tokyo, Japan) to close large colorectal mucosal defects. We also pro-
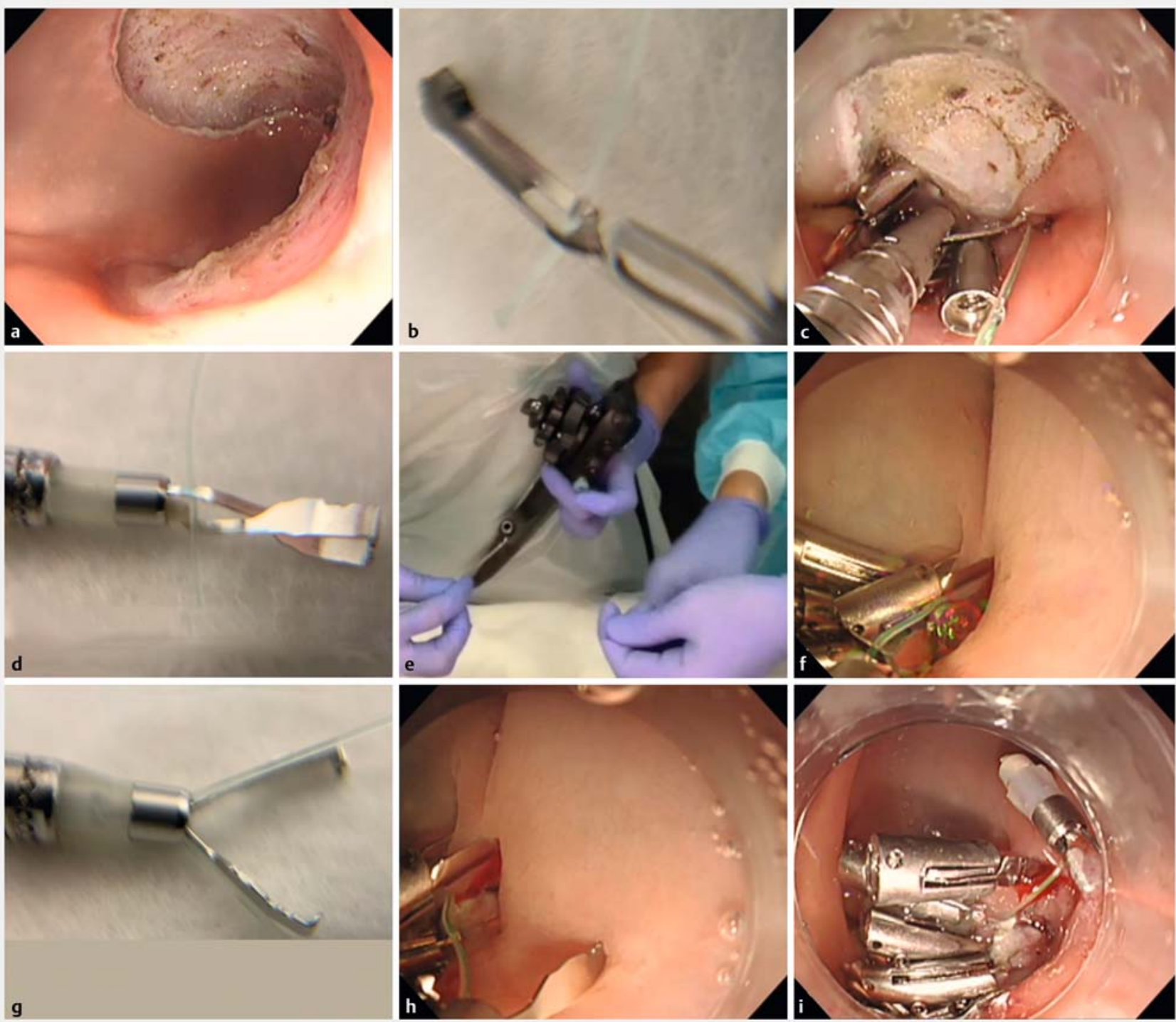

- Fig. 1 Example use of novel method for closure of large defects after endoscopic submucosal dissection (ESD) showing: a a 70-mm mucosal defect with a circumference approximately three-quarters that of the intestinal tract; $\mathbf{b}$ the reopenable clip over line method (ROLM), which involves the line coming out of the endoscope accessory channel being threaded through one hole of a 16-mm reopenable-clip; $\mathbf{c}$ the mucosal defect gradually becoming smaller with repeated use of the ROLM; $\mathbf{d}-\mathbf{f}$ the external end of the line being passed through the slightly opened clip, before its insertion through the accessory channel, meaning that, when the clip is in position, the line has already passed through the gap between the teeth at the base of the clip; $\mathbf{g}, \mathbf{h}$ the modified locking-clip technique (M-LCT) being performed; $\mathbf{i}$ the final appearance after pulling the external line coming out of the endoscope accessory channel to cut the line at the base of the clip and leave a completely closed mucosal defect. 


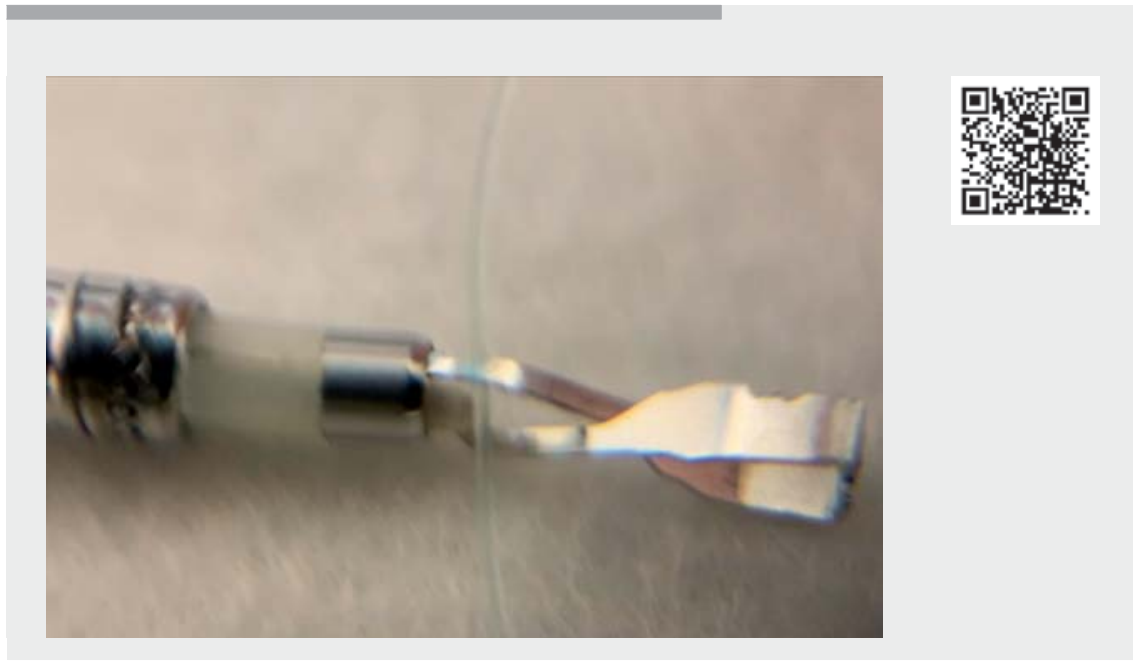

$\checkmark$ Video 1 Large mucosal defect closure in the sigmoid colon using the reopenable clip over line method and the modified locking-clip technique.

posed a method of fixing the line to the first and second clips after the mucosal defect had been closed with threadassisted mucosal defect closure using the locking-clip technique (LCT) [3]. However, LCT is difficult technically because it requires fixation by threading a line through the gap between the teeth at the base of the clip (EZ clip, HX-610905; Olympus) in the intestinal tract [4]. Therefore, we proposed a modified LCT (M-LCT) that involves performing the LCT by inserting the line through the gap between the teeth at the base of the clip before it is passed through the accessory channel of the endoscope.

- Video 1 shows the closure of a large mucosal defect in the sigmoid colon using the ROLM and M-LCT ( Fig. 1; - Video 1). The patient had a 65-mm tumor in the sigmoid colon and was left with a post-ESD mucosal defect of $70 \mathrm{~mm}$. We used the ROLM to close the mucosal defect, fixed the line to the clip with the M-LCT, and cut the line at the base of the clip. The procedure was successful and the patient was discharged without any adverse events.

Use of the ROLM and the M-LCT is a novel method for reliably closing large colorectal mucosal defects.
Endoscopy_UCTN_Code_TTT_1AQ_2A]

\section{Competing interests}

The authors declare that they have no conflict of interest.

\section{The authors}

Tatsuma Nomura ${ }^{1,2}$, Shinya Sugimoto ${ }^{2}$, Mayu Kawabata ${ }^{2}$, Jun Oyamada ${ }^{2}$, Akira Kamei $^{2}$

1 Department of Gastroenterology, Mie Prefectural Shima Hospital, Shima, Mie, Japan

2 Department of Gastroenterology, Ise Red Cross Hospital, Ise, Mie, Japan

Corresponding author

\section{Tatsuma Nomura, MD}

Department of Gastroenterology, Mie Prefectural Shima Hospital, 1257 Ugata, Ago, Shima, Mie 517-0595, Japan m06076tn@icloud.com

\section{References}

[1] Pohl H, Grimm IS, Moyer MT et al. Clip closure prevents bleeding after endoscopic resection of large colon polyps in a randomized trial. Gastroenterology 2019; 157: 977984

[2] Kato M, Takeuchi Y, Yamasaki Y et al. Technical feasibility of line-assisted complete closure technique for large mucosal defects after colorectal endoscopic submucosal dissection. Endosc Int Open 2017; 5: E11-E16

[3] Nomura T, Kamei A, Sugimoto $S$ et al. Thread-assisted mucosal defect closure using the locking-clip technique after gastric endoscopic submucosal dissection. Endoscopy 2018; 50: E342-E343

[4] Nomura T, Kamei A, Sugimoto S. Clip line traction method using locking-clip technique for colorectal endoscopic submucosal dissection. Dig Endosc 2019; 31: E72-E73

\section{Bibliography}

Endoscopy 2022; 54: E63-E64

DOI 10.1055/a-1381-6435

ISSN 0013-726X

published online 5.3.2021

(c) 2021. Thieme. All rights reserved.

Georg Thieme Verlag KG, Rüdigerstraße 14, 70469 Stuttgart, Germany

\section{ENDOSCOPY E-VIDEOS}

https://eref.thieme.de/e-videos

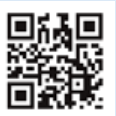

Endoscopy E-Videos is an open access online section, reporting on interesting cases and new techniques in gastroenterological endoscopy. All papers include a high quality video and all contributions are freely accessible online. Processing charges apply (currently EUR 375), discounts and wavers acc. to HINARI are available.

This section has its own submission website at https://mc.manuscriptcentral.com/e-videos 